\title{
LIBRI RICEVUTI E RECENSIONI
}

\section{Libri ricevuti.}

P. J. Bulmax, G. S. Hobson and B. C. Taylor: Transferred Electron Devices. Academic Press, London and New York, 1972; p. X.402; $£ 8.50$.

B. H. VAssos and G. W. EwIxg: Analog and Digital Electronics for Scientists. WileyInterscience, a division of John Wiley and Sons, Ine., New York, London, etc., 1972; p. X-405; $£ 5.50$.

J. H. Crawford jr. and L. M. Slifkix, Editors: Point Defects in Solids. - Vol. I: General and Ionic Crystals. Plenum Press, New York, London, 1972 ; p. Xv.556; \$43.00. F. HuND: Geschichte der physikalischen Begriffe. Bibliographisches Institut, Mannheim, Wien, Zürich, 1972; p. 410; DM. 9.90.

T. E. FABER: Introduction to the Theory of Liquid Metals. Cambridge at the University Press, 1972 ; p. XIII-587; £ 12.00 net.

H. Kangro, Editor: Planck's Original Papers in Quantum Physics. Taylor and Francis, Londoll, 1972 ; p. VIII-60; $\mathfrak{f} 70$.

C. H. BAMFord and C. F. H. TIPPER, Editors: Comprehensive Chemical Kinetics. Vol. 10: Ester Formation and Hydrolysis and Related Reactions. Elsevier Publishing Company, Amsterdam, London, New York, 1972; p. X-309; Dfl. 95.00.

L. VAN Hove: High-Energy Physics 25 Years After the Discovery of the $\pi$ Meson. CERN, Geneva, 1972; p. v.29; s.i.p.

R. BUdde, G. Ekspong and S. O. Holmgren, Editors: European Spiral Reader Symposium, Stockholm, May 30-June 1, 1972. CERN, Geneva, 1972; p. vi-310; s.i.p

\section{Recensioni.}

R. C. Haymes - Introduction to Space Science. John Wiley and Sons, Inc., New York, London, etc., $1971 ;$ p. XVII-556; $£ 6.75$.

Con il rapido evolversi delle scienze spaziali era inevitabile che qualche ricercatore e, allo stesso tempo, insegnante presso un'università scrivesse un libro di testo sullo spazio.

Solitamente chi lavora in un campo ed insegna la stessa materia in un corso tende a presentare una visione quanto meno personale dell'argomento, talvolta trascurando altri aspetti che sono tutt'altro che di second'ordine.

Haymes, raccogliendo le annotazioni del corso da lui tenuto alla Rice University, ha, forse, temuto di incorrere in questo preciso difetto e, per eccesso di zelo, ha, ci sembra, ecceduto dalla parte opposta. Non solo nel volume i raggi $X$, argomento di ricerca dell'autore, non sono la parte centrale del testo, ma sono quasi trascurati.

Inoltre gli argomenti trattati sono cosi numerosi che diventa arduo anche per il nostro, che pur sa essere conciso e 